\title{
Balance de la producción académica sobre participación política de las mujeres en colombia 2000-2019*
}

\author{
Balance of academic production on political participation \\ of women in Colombia 2000-2019
}

María Andrea Palta Limas
Juliana Rodríguez Arango
William Darío Chará Ordóñez
****

Rec: 08/08/2019

Acep: $27 / 05 / 2020$

\section{Resumen}

El presente artículo tiene como objetivo dar cuenta de la producción de tipo académico realizado en relación con la participación política de las mujeres en Colombia. Se llevo a cabo un análisis documental mediante la revisión sistemática de producción académica publicada en repositorios institucionales y de consulta académica a partir de criterios de inclusión como: participación política de las mujeres, antecedentes de participación política, escenarios en los cuales las mujeres participan, marcos normativos, alcances institucionales y retos todavía presentes para las mujeres que quieren lograr un posicionamiento en escenarios de toma de decisión. El resultado permite evidenciar que la evolución de la participación política de las mujeres, caracterizada en primera medida por su total exclusión de los espacios de opinión y toma de decisión, hasta su consideración como sujetos de derechos en el sistema internacional, ha permitido que actualmente exista un respaldo normativo, tanto a nivel internacional como nacional, que, si bien es débil, en Colombia resaltan casos replicables.

* Este artículo de investigación es producto del proyecto de investigación denominado "Construcción de Paz y tramitación de conflicto en el departamento del Cauca 2018: una mirada territorial y con enfoque diferencial" adscrito a Grupo de Investigación Giaprip de la Universidad del Cauca y ejecutado en asocio por el grupo GIISCH de la Corporación Universitaria Autónoma del Cauca, Colombia.

** Investigadora del programa de Ciencia Política de la Universidad del Cauca, Colombia. Investigadora del Semillero LVMEN adscrito al Grupo de Investigación en Actores, Procesos e Instituciones Políticas (Giaprip) del programa de Ciencia Política de la Universidad del Cauca. Popayán, Colombia. Correo electrónico: andreapalta@unicauca.edu.co; Orcid: https://orcid.org/0000-0002-9001-5913

*** Investigadora del programa de Ciencia Política de la Universidad del Cauca, Colombia. Investigadora del Semillero LVMEN adscrito al Grupo de Investigación en Actores, Procesos e Instituciones Políticas (Giaprip) del programa de Ciencia Política de la Universidad del Cauca, Popayán, Colombia. Correo electrónico: rjuliana@unicauca.edu.co; Orcid: https://orcid.org/0000-0002-6644-3205

**** Politólogo de la Universidad del Cauca, Colombia, magíster en Sociología de la Facultad Latinoamericana de Ciencias Sociales (Flacso) sede Ecuador. Docente investigador del programa de Derecho y coordinador del Semillero de Investigación en estudios de Conflicto, Derechos Humanos y Justicia Transicional, adscrito al Grupo de Investigación Interdisciplinar en Ciencias Sociales y Humanidades (GIICSH) de la Corporación Universitaria Autónoma del Cauca, Popayán, Colombia. Correo electrónico: william.chara.o@uniautonoma.edu.co; Orcid https://orcid org/0000-0002-7558-2047 
Palabras clave: mujeres, participación política, participación política de mujeres, enfoque de género, construcción de paz.

\begin{abstract}
This article aims to account for academic production carried out in relation to the women's political participation in Colombia. The documentary analysis was carried out through systematic review of academic production published in institutional repositories and academic consultation based on inclusion criteria such as: women's political participation, political participation backgrounds where women participate, regulatory frameworks, institutional scope and challenges still present for women who want to have a position in decision-making scenarios. Results shows that the evolution of women's political participation, characterized in first place by their total exclusion from spaces of opinion and decisionmaking and reaches their consideration as subjects of rights in the international system. This has allowed for there to currently exist a normative support, both internationally and nationally, which, although weak, may be highlighted with replicable cases in Colombia.
\end{abstract}

Keywords: women, political participation, women's political participation, gender approach, peace building.

\section{Introducción}

En una sociedad como la colombiana, caracterizada por una estructura de poder que propicia el escenario de mejores condiciones para los hombres por encima de las mujeres en la esfera de lo público, relegándolas al ámbito de lo privado desde las llamadas sociedades tribales, se puede decir que el acelerado progreso político, social, humano y económico del país ha ido acompañado por su contrario: un lento proceso de inmersión de la mujer en la actividad política y los altos cargos de administración pública aun cuando esta ha sido históricamente promotora de procesos de construcción de paz, al igual que lideresa de la consecución de progreso y desarrollo.
Lo anterior hace parte de las contradicciones de la participación social y política femenina que denota la existencia grandes retos y dificultades para alcanzar la llamada "representación paritaria", pues el enfoque de género presente en las agendas del gobierno y los acuerdos de paz del 2016 no son suficientes para evitar la invisibilización de los factores que causan la reproducción de las brechas entre hombres y mujeres, pero pueden convertirse en oportunidades para su reducción, que, según la Asamblea de las Naciones Unidas del 2011 "siguen estando marginadas en gran medida de la esfera política en todo el mundo, a menudo como resultado de leyes, prácticas, actitudes y estereotipos de género discriminatorios" (ONU, 2012).

En Colombia, durante décadas las mujeres estuvieron excluidas de procesos decisorios del país, sin embargo, distintos esfuerzos para promover el liderazgo y la representación política han sido énfasis de la acción estatal, de la mano de la cooperación internacional y los movimientos sociales que han evidenciado y problematizado la baja presencia de las mujeres en los escenarios de decisión política y la importancia de generar cambios culturales más que normativos que incentiven el empoderamiento de las mujeres y propicien los espacios para su inserción en la esfera pública. Lo anterior lo reafirma el Observatorio de Igualdad de Género de la Comisión Económica para América Latina y el Caribe (Cepal) que plantea que, aunque actualmente América Latina y el Caribe cuenta con mujeres en altos cargos de Administración Pública (5 presidentas en total), el número de legisladoras, juezas y alcaldesas no supera el 26 \% en promedio. (Cepal, 2014).

Con base en esta descripción general de la participación política de las mujeres en América Latina y en especial en Colombia, emerge la inquietud de indagar por las maneras en las que se ha investigado el tema, sus avances y modos de comprensión. En ese sentido, este artículo tiene como objetivo dar cuenta del desarrollo de la producción académica 
realizada alrededor del tema de la participación política de las mujeres en Colombia a partir de la comprensión de la evolución de la participación política y su desarrollo normativo.

\section{Metodología}

Debido al anterior objetivo, el diseño metodológico seleccionado para tal fin se ampara en una metodología cualitativa de corte documental a manera de revisión sistemática de literatura de aquellas investigaciones realizadas sobre participación política de mujeres en Colombia. Como criterios de inclusión en la revisión se consideraron las siguientes palabras clave: participación política de las mujeres, antecedentes de la participación política de mujeres y marco normativo de la participación.

Con estos criterios en mira, la búsqueda de la producción académica se adelantó en los repositorios Istor, Ebsco y Proquest con una intencionalidad: evidenciar la basta producción académica y de calidad indiscutible que, por motivos de indexación, no se encuentra referenciada en repositorios digitales considerados principales. Además, se consultaron repositorios institucionales de distintas universidades colombianas: Pontificia Universidad Javeriana, Universidad del Valle, Universidad de Manizales, Universidad del Rosario, Universidad Militar Nueva Granada, Universidad Pedagógica, Universidad Católica, Universidad Autónoma del Caribe. En todas las consultas la ventana de observación de la producción académica contempló el periodo 2000-2019.

\section{Resultados}

El primero de los hallazgos en este diseño metodológico evidencia que la producción académica sobre participación política y sus temas derivados se encuentran en mayor medida desde el 2014 hasta el 2018 (figura 1). El 2016 fue el año con mayor número de publicaciones (16,67\%), lo cual devela que estos temas toman mayor fuerza desde la firma del Acuerdo Final para la Terminación del Conflicto y la Construcción de una Paz Estable y Duradera, en particular, con los avances de la mesa de subcomisión de género que se convierte en un referente mundial en el que las mujeres se posicionan en la agenda pública y también en el desarrollo de actividades que garanticen su participación en política.

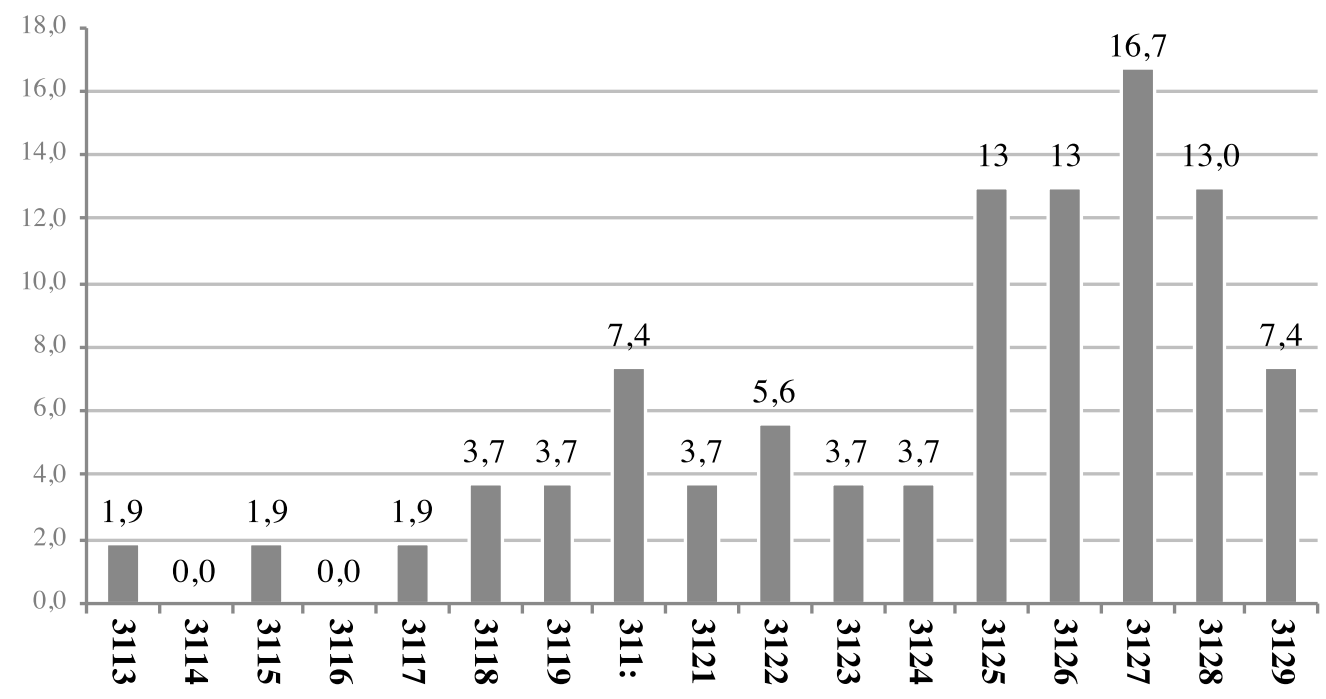

Figura 1. Porcentaje de publicaciones en Colombia por año Fuente: elaboración propia a partir Jstor, Ebsco, Proquest y repositorios digitales institucionales 
Por ubicación geográfica se privilegian los escenarios de estudio de carácter regional; se concentran en las ciudades capitales más que en las intermedias (figura 2). Tan solo el $19,7 \%$ de las publicaciones consultadas hacen mención a estudios de carácter nacional.
Esto permite indicar la necesidad de continuar con investigaciones que prioricen en resaltar los casos regionales en los que se pueden encontrar aquellos casos que permitan una comprensión más específica del tema que a esta investigación ocupa.

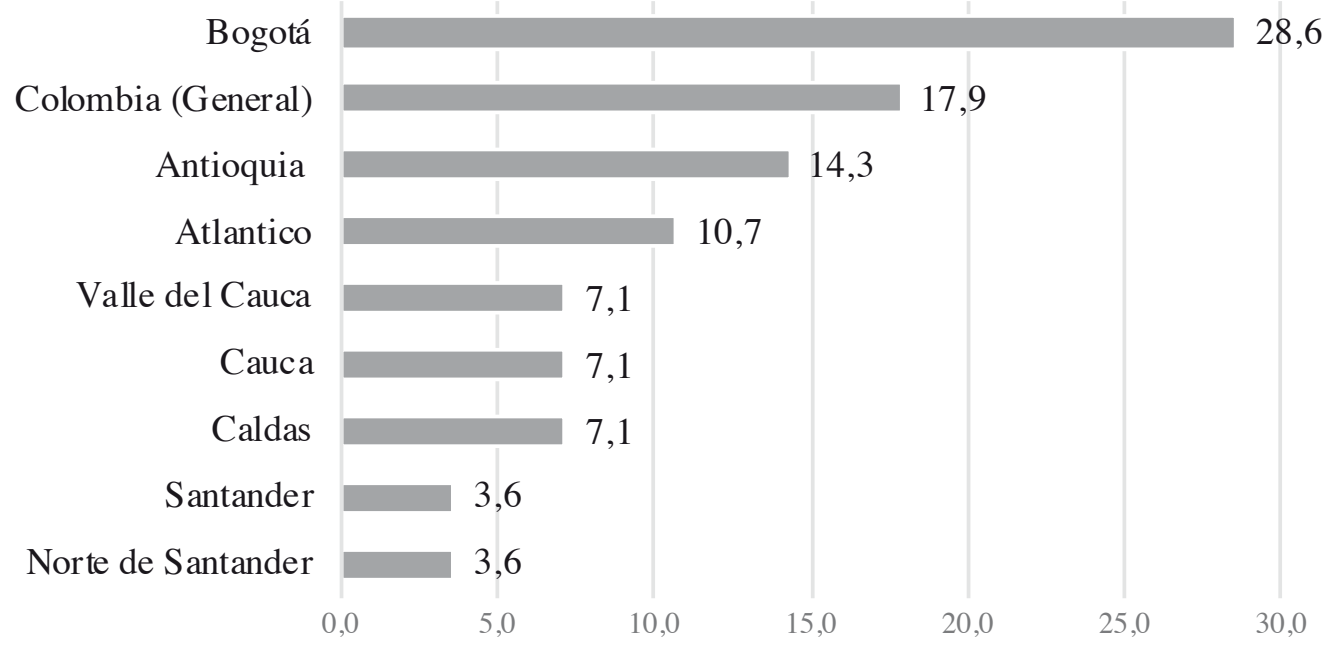

Figura 2. Porcentaje de publicaciones por ciudad

Fuente: elaboración propia a partir de Jstor, Ebsco, Proquest y repositorios digitales institucionales

\section{Antecedentes y alcances normativos que posibilitan la participación política de las mujeres en Colombia}

La búsqueda del reconocimiento y posicionamiento de las mujeres en las decisiones políticas a lo largo de la historia colombiana se ha logrado, entre otras cosas, gracias a la forma en que se organizaron para obtener avances en un marco normativo progresista que las ampare, con la formulación de diferentes decretos, normas y demás herramientas jurídicas que, en su momento, han representado logros significativos para las mujeres en su papel como ciudadanas políticas.

Gutiérrez (2002) plantea que gran parte de las mujeres han llegado a la política más fácilmente por la vía de designación de cargos que por la vía electoral, esto en parte porque muchas de las iniciativas de inclusión de la mujer no están respaldadas por una real voluntad de los partidos políticos ni del Estado. Por ejemplo, la adopción de leyes de acción afirmativas para la inclusión de las mujeres en la política fueron aceptadas por presión internacional, debido a que los demás países latinoamericanos habían incluido dentro de sus agendas el tema de la paridad política entre hombres y mujeres.

El postulado mencionado que indica que la participación de las mujeres ha estado supeditado a cargos nominales encuentra un soporte conceptual basado en lógicas patriarcales históricas en Colombia que se continúan reproduciendo con miras a mantener la figura determinante de los hombres en los procesos decisorios. Al respecto, Bernal (2006) afirma que normalmente en el campo político sobresalen y ganan los comportamientos, actitudes y valores tradicionalmente considerados como masculinos. En lo que respecta al campo de la competencia electoral argumenta que reglas como el voto preferente, el umbral, y la fórmula d'Hondt resultan negativos para las mujeres y para las minorías políticas porque a 
través de estas se refuerzan barreras previas que, aunque no son de tipo formales, pueden operar para excluir a nuevas y/o nuevos actores políticos..

Además, Bernal (2006) afirma que las mujeres logran mejores resultados en circunscripciones de siete o más curules, ya que cuentan con mayores oportunidades de ser incluidas en las listas debido a que los partidos pueden asumir el riesgo de presentar nuevas candidaturas, que, en ocasiones, son de mujeres. Respecto a los partidos políticos, sostiene que a menudo, estos son mejores para idear políticas de incorporación de las mujeres en las posiciones internas del partido que para postularlas como candidatas y que, por lo general, sus esfuerzos en la labor de incentivar la paridad entre hombres y mujeres son mediocres.

A partir de esta contextualización, una revisión de antecedentes de carácter normativo resulta pertinente para comprender la participación política de las mujeres. Para este objetivo, la investigación de Murray (2009) que presenta un análisis de los procesos de participación electoral y política en la época de la Gran Colombia y que mediante la revisión y análisis de unas cartas escritas por mujeres dirigidas al general Mosquera da cuenta de que la participación política de las mujeres se establece por factores de asociación entre ellas y las redes sociales establecidas alrededor del general en el momento de su rebelión contra el gobierno de Mariano Ospina. En su gran mayoría, las cartas están dirigidas con el fin de comunicar asuntos de política, sobre la guerra civil y de interés general.

La principal característica de las cartas de dichos años (1859-1862) estriba en los diferentes lugares de origen. Aunque algo más de la mitad de las cartas escritas por las mujeres (36 o casi 51\% del total) procedían del Gran Cauca e incluye ciudades mayores como Popayán, Pasto y Cali. Esto permite colegir que el papel de la mujer fue clave en procesos de incidencia política, pero carecía de representatividad política. Las decisiones no las tomaban ellas, aunque sí se soportaban en la evidencia de sus escritos.

En esta línea de análisis del rol de la mujer en escenarios de carácter político, Machado (2018) plantea el papel de la mujer en la preparación ideológica del pensamiento emancipador neogranadino, hace énfasis en la actividad de la mujer en las luchas independentistas y el ejemplo que dieron las más destacadas heroínas al involucrarse como coparticipantes en la lucha por la conquista de la independencia. Las mujeres se fueron integrando de tal forma que hicieron parte de la dirección de tropas y lograron desdibujar, en cierta medida, los mitos existentes acerca de su desempeño en labores de alto cargo y los que sus decisiones tuviesen igual incidencia.

Patiño (2011) explica que el Estado colombiano y diversas organizaciones han generado procesos de participación para las mujeres y desarrollado políticas públicas que favorecen su integración en la escena pública con la creación de diversas herramientas. Entre ellas destacan la política de reactivación social, enfocada en la contribución de igualdad entre hombres y mujeres, y el Acuerdo Nacional por la Equidad entre mujeres y hombres. Pero también es cierto que estas políticas no son suficientes para darle una mirada más profunda al problema de la inequidad en la participación, esto debido a que la mayoría de dichos esfuerzos están orientados hacia la consecución de igualdades, que, como se mencionó anteriormente, son difíciles de alcanzar y generan aún más inequidad y desconocimiento de los seres humanos como sujetos diferenciados y capaces de reconocerse en la sociedad.

Así las cosas, surge la necesidad de aclarar las diferencias de género existentes en liderazgo. Ruiloba (2013) plantea una discusión sobre la controversia debido a la evidencia científica de que existen diferencias de género en el liderazgo que se han prolongado durante más de un siglo. Esto por medio 
de plantear preguntas que enmarcan los resultados con variables tales como la perspectiva de género. En este aspecto nota ciertas características de las mujeres que acceden al poder político en un alto nivel, en el cual suponen una aristocracia femenina en los círculos masculinos, son una elite dominada en el campo político que dentro de los partidos rigen a la oligarquía masculina.

Según Ruiloba (2013), Las mujeres se convierten en una elite aislada entre el poder masculino y la masa femenina, finalmente las denomina una elite problematizada por su tensión entre lo profesional versus lo femenino. Esto deja en claro que con frecuencia la vida pública tiene un mayor precio para las mujeres, por lo que las esferas más altas de poder siguen en manos de los hombres.

El panorama Latinoamericano no es en gran parte el más favorable para las mujeres. En el continente, los limitados escenarios de participación en la toma de decisión política han llevado a que las mujeres establezcan nuevas formas de organizarse para acceder a estos escenarios de participación. Para Dietrich (2014), una de las formas en las que las mujeres se lograron organizar en América Latina fue hacerse miembros de grupos insurgentes. En ellos, las maneras en que las mujeres se organizan se moldean al orden interno para alcanzar las metas de carácter político, sin embargo, dentro de estas organizaciones continúa la reproducción de lógicas patriarcales que hacen que sigan las asimetrías de poder en contra de las mujeres. Lo anterior lleva a que aún la exclusión hacia las mujeres se encuentre presente en la lucha armada.

Ahora bien, al tomar en consideración este panorama de América Latina e iniciar con los antecedentes en Colombia es necesario rescatar la investigación de Vos (2004); a partir de un balance histórico desde la década de 1920, da cuenta de la participación y ciudadanía de las mujeres en Colombia y destaca el liderazgo femenino en la lucha por la construcción y fortalecimiento de los procesos democráticos. Vos (2004) afirma que la historia de las mujeres representa solo una mirada fémina y un modo nuevo de hacer historia, seguido de los avances en materia de derechos humanos, enmarcados por hechos históricos que dan cuenta de los primeros procesos organizativos de las mujeres, tales como el Congreso Femenino realizado en Bogotá; data que la revolución feminista inicia en estos años, pasa luego por un periodo de modernización del Estado y el rol de las mujeres.

En términos del desarrollo normativo colombiano está la investigación de Lozano y Molina (2014) que se apoya en los postulados de Richard Posne para quien la racionalidad del comportamiento de los individuos y la eficiencia de las normas jurídicas como mecanismos de incentivos -que al ser traducidos a precios se convierten en los incidentes de conductas- centran su atención en la eficacia y eficiencia de la aplicación del artículo 28 de la Ley 1437 de 2011. Parte de un abordaje de los antecedentes en los cuales resaltan que, en el contexto globalizado, las mujeres siempre han sido un grupo marginado y es solo por las luchas que han adelantado que lograron que el Estado escuche las múltiples necesidades de las mujeres y sus derechos no sean cada vez más vulnerados.

En ese sentido, para Lozano y Molina (2014) uno de los primeros actos reivindicativos hacia las mujeres es el Acto Legislativo No. 3 de 1954 durante el gobierno del General Gustavo Rojas Pinilla, un momento histórico clave. Es entonces cuando por primera vez se permite que las mujeres ejerzan su derecho al voto para la Presidencia de la República. Esto se convirtió en una plataforma de participación que posteriormente permitió que trece mujeres ocuparan gobernaciones en el país y, además de ello, logró la expedición del Decreto 2028 de 1974 (Estatuto de Igualdad de Género) que trajo consigo el avance de tipo normativo, producto de las luchas que adelantaban las mujeres en materia de derechos humanos. Posteriormente, esto se reflejó 
en la Convención sobre la Eliminación de Todas las Formas de Discriminación Contra la Mujer adoptada por la Asamblea General de las Naciones Unidas con el Decreto 1398 de 1990.

De esta manera quedó reglamentado el panorama para la participación de las mujeres en los procesos de la constituyente de 1991. Después de arduas jornadas y luchas que duraron más de nueve años, se consiguió la aprobación de la Ley 581 de 2000, la cual tiene por objeto promover la participación efectiva de la mujer en los niveles de poder público. Esta ley consagra la cuota mínima del 30 \% en el desempeño de cargos, tales como los de máximo nivel decisorio y en los otros niveles decisorios (Ley 581 de 2000). La Constitución Política de 1991 es el eje articulador que viabiliza la aplicación de la Ley 581 de 2000 y el artículo 28 de la Ley 1437 de 2011, de modo que la convierte en una medida eficiente para aumentar paulatinamente la participación política y electoral de la mujer.

Es en este momento en el cual las relaciones juegan un papel importante, que, sin embargo, continúan con la reproducción de lógicas que favorecen a hombres y dejan en desventaja a las mujeres en casos de representatividad y de visibilización, tal como lo afirma Hernández (2014). Hernández (2014) evidencia la relación que tiene la prensa con el desarrollo de la cotidianidad de las mujeres, sostiene la hipótesis de que la prensa da cuenta del lugar real que tienen las mujeres en la sociedad, más allá de una política pública y del imaginario de una existencia de igualdad de género. Es por ello que como parte de los hallazgos plantea que es necesario trabajar en procesos de cambio cultural. Además, que la prensa sobre las mujeres se basa en el reconocimiento y funciona como garante para que los procesos de participación política que adelantan sean fielmente comunicados sin desvirtuar lo que ellas hacen solo por el hecho de ser mujer.

Un claro ejemplo del devenir histórico de los procesos de trasformación de la participación femenina se encuentra en el texto de Acevedo y Uribe (2015) que afirma que han transcurrido más de doscientos años desde los eventos que dieron origen a la Revolución Francesa y que con esta surgió un nuevo orden político que originó nuevas ideas y configuraciones en el gobierno. Se generaron voces a favor y en contra de las mujeres debido al reconocimiento de su ciudadanía en el Estado y por ser consideradas sujetos políticos. Los intereses que las movían eran la vocería y representación del ámbito privado. Es así como «las ideas desarrolladas por Voltaire, Rousseau, Montesquieu, Diderot, Adam Smith, John Stuart Mill, John Locke y Hobbes, entre otros, hicieron posible la configuración de este nuevo orden político conocido como Estado liberal o de derecho", lo que se convierte en el escenario global que permite comprender cómo las representaciones mentales presentes en una sociedad determinan o influyen en procesos políticos, en este caso los de las mujeres.

Al retomar la mirada hacia América Latina y el caso particular de Colombia, se encuentra que Tula (2015) recoge dicho argumento al aseverar que, en relación con la apertura de mecanismos de participación efectiva de las mujeres en la política, se ha contado con la intervención de varios organismos a través del diseño y establecimiento de una ley de cuotas de género.

Tula (2015) plantea que resulta importante una discusión sobre la necesidad de que exista una discriminación positiva para lograr que las mujeres tengan espacios en estos escenarios, toda vez que, lo que realmente se ha logrado es que las mujeres sean colocadas en lista con el fin de cumplir con lo establecido por la norma. Lo anterior va en contravía de una representatividad real que permita una cooperación entre hombres y mujeres para lograr transformaciones significativas. El esbozo de su propuesta plantea dos alternativas. La primera está relacionada con la paridad política como solución para los problemas que ha dejado la implementación de la ley de cuotas de género. La segunda sugiere 
mantener la vigencia del Acto Legislativo 01 de 2003, por el cual se admite que los partidos opten por listas cerradas y bloqueadas o voto preferente para así lograr equilibrar el posicionamiento de los candidatos y las candidatas y afianzar las expectativas de acceso a la representación.

En clave de esta última propuesta, resulta importante lo que plantea Gutiérrez (2015) quien menciona que a pesar de que la mayoría de los partidos disponen

de unidades de la mujer, estas tienen una muy limitada participación y poca influencia en las decisiones partidarias. Menos de la mitad de los partidos cuenta con representación directa en su cuerpo ejecutivo y menos de un tercio participa en la formación de listas de candidatos o en la elaboración de planes de gobierno. A su vez, solo un 15 por ciento de los partidos asigna partidas regulares a las unidades de la mujer para su funcionamiento orgánico, situación que limita su capacidad para diseñar e implementar estrategias y acciones a largo plazo dirigidas a promover la igualdad de género. Así entonces, la oferta electoral de los partidos es poco balanceada en términos de sexo: en promedio solo uno de cada cuatro candidatos es mujer. Asimismo, solo un $14 \%$ de las listas fueron encabezadas por mujeres.

Al indagar por las limitaciones de la implementación de las cuotas políticas, Lora (2015) plantea que en Colombia el obstáculo de fondo que impidió la introducción de cuotas políticas para la mujer residió en el propio sistema político imperante en el país y, en particular, en sus dos ejes estructurales: el sistema electoral y el régimen de partidos políticos.

Según Lora (2015), para que finalmente se diera existencia a las cuotas como medidas temporales de acción afirmativa de obligatorio cumplimiento, se tuvieron que adelantar sucesivas reformas políticas de carácter constitucional durante el transcurso de dos décadas. Del mismo modo, afirma que para determinar la eficacia de las cuotas políticas femeninas en la superación de la subrepresentación política de la mujer -eficacia que la teoría les otorga- y vislumbrar si su futuro era o no promisorio, fue necesario conocer en qué medida las reformas políticas de los sistemas electoral y de partidos políticos lograron remover de forma efectiva las barreras de diversa índole que histórica y estructuralmente han perpetuado el ostracismo político de las mujeres colombianas.

Como conclusión de ello, Lora (2015) afirma que luego del difícil y largo trámite que dio como resultado 134 cuotas de participación política para las mujeres, fueron más la presión de instancias internacionales que las exigían en los pactos, convenciones y otros instrumentos de protección para las mujeres y suscritos por el país y la permanente presencia a través de la movilización y cabildeo del movimiento de mujeres, que la iniciativa y promoción consciente de la política de género de la totalidad de los partidos. Esto a su vez indica solo un cambio de forma, aunque determinante en la remoción de barreras para subsanar la precaria representación política de las mujeres colombianas.

De manera particular y como ejemplo de esta línea argumentativa, la investigación de Uribe (2015) da cuenta de la representación histórica de la participación política de las mujeres de los cuerpos legislativos de Santander. Afirma que "durante los siglos XIX y XX, y a medida que la universalidad del sufragio se fue imponiendo por la acción de los diversos grupos de presión, la representación política siguió siendo tema de discusión, debate y producción teórica" (Uribe, 2015, p. 23). Es por lo que se establece que la cooptación de las dirigentes femeninas por parte de los partidos políticos más el reflujo del movimiento feminista dificultaron la construcción autónoma de formas y prácticas distintas de hacer política.

Lo anterior guarda concordancia con el planteamiento de Ariza (2015) quien afirma que existe una dinámica bajo la que las mujeres tienen 
derecho a la participación política, pero que esto no implica que se configure una verdadera realidad de participación, sino que se limita a unas condiciones de representatividad. Estas condiciones, sumadas a un sistema social de carácter patriarcal, hacen que las condiciones de vida de las mujeres se vean ampliamente problematizadas en el país.

Aquello se debe a factores de tipo cultural, Beltrán (2016) plantea que las mujeres que tienen la cualidad de ser esposas y madres en el ámbito privado quedan excluidas del estatus de individuo y por lo tanto de la participación en el ámbito público, porque desde la perspectiva liberal constituida con nociones patriarcales la participación en la esfera pública se rige por los criterios de la igualdad, los derechos y la propiedad privada, que solo son aplicables de manera universal, impersonal y convencional a los hombres. Así entonces, ampliar los principios de participación en la esfera pública a las mujeres con la ciudadanía sin provocar una ruptura de la estructura patriarcal de la vida privada es pretender que solo se logre la plena e igual participación de las mujeres en la vida pública sin que se produzcan cambios en la esfera doméstica.

Con respecto a la Ley de cuotas de género Triana (2016) señala que esta se establece en Colombia y fomenta la participación femenina en las circunscripciones grandes y medianas que por sus características, facilitan la obtención de escaños para las mujeres. No obstante, se dejan de lado en las circunscripciones en las que los partidos políticos tienen menores incentivos para incluir a las mujeres en sus listas y por lo tanto, estas tienen menores posibilidades de ser elegidas. Así, en más de la mitad $(57,57 \%)$ del territorio nacional, en el que se elige a casi un tercio (29,51 \%) de los representantes a la Cámara, no aplican los efectos de la cuota mínima de género.

El alcance que tiene la cuota de género en relación con el aumento de la participación política femenina es limitado, pues las condiciones del sistema electoral no son las más favorables para la implementación de la cuota. La inaplicación de esta en una buena parte de las circunscripciones electorales, el carácter opcional del uso de listas cerradas y la ausencia del mandato de posición son elementos que contribuyen a reducir el número de mujeres electas, en especial en la Cámara de Representantes.

A pesar de la existencia de esta ley, hay una serie de barreras culturales que dificultan la participación de las mujeres. Estas se basan en la dominación masculina, fenómeno estructural que se expresa en estereotipos de género relacionados con la familia, la belleza y la feminidad. Al estar estos presentes en todas las interacciones sociales impiden el acceso de las mujeres al campo político y por lo tanto, son una restricción para el adecuado funcionamiento de la cuota. En especial porque la presencia de dichos estereotipos evita que las mujeres tengan una formación lo suficientemente buena como para entrar a competir en un campo tradicionalmente masculino y a su vez, frena sus candidaturas por la falta de financiación equitativa para sus campañas.

Entre los resultados que deja la implementación de la ley de cuotas, Morales (2016) afirma que las mujeres en Colombia tienen un $23 \%$ de participación política, es decir, sigue siendo precaria su participación política en el Senado a pesar de la ley de cuotas efectuada para la equidad en participación. En este aspecto, la mujer no alcanza ni el 30 \% de representación como sí ocurre en otros países latinoamericanos como Argentina y México. Cabe destacar que esta ley en Colombia no garantiza el 30 \% de participación de la mujer en la rama legislativa, es decir, en los cargos de elección. El porcentaje del 30 \% se da a nivel general en las tres ramas del poder público (ejecutivo, legislativo y judicial). A diferencia de Colombia, en países como Argentina y México la mujer tiene asegurado el 30 \% en el Senado, lo cual permite un empoderamiento de las mujeres en las elecciones parlamentarias debido al número de cuotas. 
Otro de los casos en los que se evidencia el débil alcance de la aplicación de la norma lo señala Rueda (2016) al afirmar que en la Unión Patriótica (UP), el único partido político legalmente constituido en el mundo que ha sufrido un proceso de exterminio y persecución política, las mujeres comenzaron a tener protagonismo en el momento en que se presentaron los asesinatos selectivos y las masacres en los lugares en los que la UP tenía gran influencia. En su mayoría, los hombres fueron las víctimas de los ataques violentos, ya que eran estos los portadores del partido en la zona. Es entonces que las mujeres se organizaron y decidieron hacer frente a este flagelo mediante procesos de recuperación de memoria adelantados años después y cuyo objetivo era evitar el olvido del fenómeno y su empoderamiento como mujeres.

Para Rueda (2016) las formas en las que las mujeres participaron se puede clasificar en cuatro categorías: 1) el contacto con las masas, en esta se hace política desde las calles; 2) la conformación de células de base en las que se establecieron proyectos que acogieran las masas; 3) la materialización del escenario de participación política, un grupo selecto cuyas acciones tenían mayor incidencia en el partido; y 4) los trabajos de la memoria realizados de la mano de organizaciones defensoras de derechos humanos (Rueda, 2016).

En este orden de ideas, Salcedo, Silvera y Garcés (2017) abordan el surgimiento de los movimientos sociales y políticos, acompañado de la consolidación constitucional del derecho de las mujeres al voto. Tienen en cuenta las perspectivas jurídica e histórica de Colombia, en las que se denota que el derecho al voto fue uno de los motivos principales para que las mujeres se organizaran y dieran paso al surgimiento de más movimientos de mujeres en Colombia, lo que incide en gran manera en la cultura política colombiana en la que la mayor parte de los cargos en política se les asignan a los hombres.
De esta manera, los movimientos y organizaciones de mujeres se establecen con más fuerza y tienen como fin la lucha por la dignidad, la reducción de la violencia y el respeto por los derechos humanos. En cierta medida, en Colombia hay un gran condicionante que lleva a que la condición sexual de las mujeres tenga como consecuencia la opresión y discriminación, lo que se convierte en un hecho que no hace excepción por clases sociales.

Un caso que permite ilustrar lo anterior lo abordan Hoyos y Nieto (2017) quienes centran su estudio en los procesos organizativos de las mujeres en el Oriente antioqueño y clarifican los significados que las organizaciones le atribuyen a la democracia. En un primer momento abordan los procesos organizativos desarrollados por mujeres tales como la interacción con organizaciones para llegar a escenarios de la esfera política. Las autoras hacen una contextualización teórica y metodológica seguida de una contextualización de las dinámicas relacionadas con la presencia de actores armados en la región y otros factores de tipo económico, así como la expansión de la industria del Valle de Aburra que ocasionó el desplazamiento de miles de familias, en su gran mayoría sacadas a la fuerza, lo cual conllevó que la confrontación armada tuviera más fuerza.

En concordancia con lo anterior, las mujeres víctimas de este flagelo se organizaron y comenzaron a actuar en función de la defensa de sus derechos, pasó de ser una organización de mujeres a convertirse en un movimiento de víctimas, esto constituye un hito histórico en su empoderamiento. Las mujeres debieron establecer cursos de acción que les permitieran tener un relacionamiento con la esfera institucional en el que los discursos de ciudadanía se fortalecieron para así lograr el reconocimiento de las mujeres en estos escenarios. Hoyos y Nieto (2017) también denotan el respaldo normativo que dicha acción tiene en la Constitución Política de 1991 y la Ley de Justicia y Paz. 
Por otra parte, Hoyos y Nieto (2017) señalan los procesos que las mujeres establecen para incidir en los espacios de decisión política, ya que han logrado que organizaciones como Asociación Provincial de Víctimas a Ciudadanas, el Centro de Acercamiento para la Reconciliación y la Reparación (CARE) y la Asociación de Mujeres del Oriente Antioqueño (AMOR) establezcan una interacción con las instituciones para así lograr su participación en la arena política formal y así ser escuchadas y que se posicionen sus reivindicaciones.

Para que lograr lo mismo que las mujeres del Oriente antioqueño es necesario conocer con detalle los elementos que deben estar presentes para el desarrollo de la participación política. Valderrama (2018) afirma que se pueden extraer tres elementos fundamentales de la idea de participación política. El primero es que la participación política se refiere a una "acción"; el segundo es que estas acciones tienen como objetivo "influir" y por último, que el espacio en donde esta acción debe ejercer su influencia es "lo político". Esta síntesis es de gran relevancia porque aclara que la participación política no se limita a la toma de decisiones sobre la Administración Pública o la estructura política de un Estado, sino que se puede entender como acciones de diferentes tipos que influyen en un espacio. Esto expande los límites de lo que se entiende por "lo político" y permite pensar en nuevas maneras de influir políticamente en la sociedad.

\section{La participación de las mujeres en Colombia}

Las mujeres en Colombia se han caracterizado por no ser partícipes de la política, pues se las ha considerado como personas con poca capacidad para toma de decisiones y/o ejecución de planes de trabajo correspondientes a la sociedad. Con base en el seguimiento realizado en el trabajo de caracterización de los hallazgos sobre la participación política de la mujer se puede resaltar la Ley de Cuotas como un logro de los procesos de luchas y resignificación adelantados por las mujeres, ya que el $30 \%$ de los cargos de la Administración Pública los deben ocupar mujeres, esto con el fin de garantizar su participación en el poder público colombiano.

En este orden de ideas, existen múltiples variables que no dejan a las mujeres participar de manera efectiva en los ámbitos sociales. Bernal (2006) resalta que existen barreras informales en el camino a la representación política por la vía electoral, se deben a que hace falta una construcción de conciencia social sobre la discriminación y subvaloración de lo femenino en todas las esferas sociales. Además de esto, identifica tres tipos de obstáculos para las mujeres en la política colombiana, los cuales dice que son permanentes, liderados e influenciados por una cultura patriarcal que conllevan la falta de oportunidades para que las mujeres entren en el ámbito político en igualdad de condiciones con los hombres. Los estereotipos prefijados sobre las mujeres dictan cómo se deben comportar y el hecho de que las mujeres no se identifiquen ni se sientan cómodas con las prácticas informales que se usan en política lleva a que prefieran rechazar una carrera política.

Londoño (2007) plantea que la exclusión de las mujeres de los escenarios de participación política formal sigue siendo una constante de la sociedad colombiana y la representación de las mujeres en los partidos políticos o en las instancias del Estado sigue siendo muy escasa, aunque se modernicen las instituciones. Se reconoce que la participación de la mujer mediante formas políticas no convencionales politizó el mundo privado y amplió el horizonte referencial de la práctica política.

Asimismo, se requiere seguimiento y veeduría de la sociedad civil y de las mujeres a los convenios y pactos internacionales firmados por Colombia para alcanzar la equidad y participación política de las mujeres, con el fin de concretar en políticas planes y proyectos específicos de obligado cumplimiento. 
esto debido a que en varias ocasiones se firman o se pactan tratados internacionales que pueden ayudar a las mujeres en las diferentes esferas, pero estos no son visibles para toda la sociedad, lo que conlleva que no se cumplan con total efectividad.

También se debe tener en cuenta que existe una fuerte relación entre la educación y la participación política, Caputto (2007) plantea el análisis de la relación existente e influencia de la educación de la mujer en Colombia y cómo esta permite configurar la participación en la vida democrática. Se desarrolla con especial atención a los antecedentes históricos que dan cuenta de las mujeres y de los procesos que han atravesado para alcanzar mayor libertad política y generan espacios de expresión y toma de decisiones.

Caputto (2007) estudió el accionar de las mujeres desde una perspectiva que prioriza las capacidades de las mujeres como elemento que permite incrementar su libertad. La autora concluye que estas se relacionan con la educación que reciben las mujeres. En esta investigación Caputto (2007) evidencia que, con la participación en escenarios de representación locales y regionales, las mujeres son escuchadas y se introducen en la política y lo político como sujetas participantes de los quehaceres públicos; denota la articulación entre mujeres y múltiples grupos u organizaciones sociales con el fin de ser escuchadas y tenidas en cuenta para la toma de decisiones.

Al indagar por los procesos de legitimización de las mujeres se encuentra, según Cortés y Parra (2008), que a través del empoderamiento de su movimiento las mujeres buscan la participación en espacios de toma de decisiones y de ejercicio del poder con miras a dar vía a procesos transformadores. Así, las formas de participación de las mujeres se enmarcan dentro del proyecto de construcción de democracia, en el cual la participación implica la posibilidad real del ejercicio de la toma de decisiones en las instancias de planeación, ejecución, fiscalización y gestión. La idea es que en ellas las organizaciones y por supuesto las mujeres que las integran y que viven un proceso de empoderamiento, puedan intervenir en los niveles de decisión política y de generación de cultura en un aspecto más amplio de transformación social (Cortés y Parra, 2008). Es decir, en palabras de los autores, la política de la mujer es uno de los grandes logros dentro de la participación femenina en ámbitos políticos y sociales, ya que representa un avance en cuanto a la equidad de género y da a conocer diferentes puntos de vista y situaciones a la sociedad. Fuentes (2009) Da cuenta de un proceso de avance en la política de equidad de género en la cual las mujeres han sido protagonistas, aquello evidencia la evolución alcanzada por las mujeres en relación con su presencia en los escenarios de toma de decisiones de tipo político.

Para la toma de decisiones efectiva se plantea -desde el deber ser- que tanto hombres como mujeres deberían tener una participación equitativa en ámbitos del poder público, cada uno de estos tiene ideas y capacidades distintas que ayudan y promueven con respecto a las necesidades de la sociedad. Herrera (2010) menciona que, pese a que la participación en el ámbito político es un derecho fundamental de hombres y mujeres con el fin de gozar de una sociedad democrática, las posibilidades de las mujeres para asumir un rol político son menores y se ven obstaculizadas por la estructura de gobierno que aún se muestra inequitativa y excluyente. Lo anterior refleja una resistencia de la clase política a la posibilidad de integrar a las mujeres como sujetas políticas, lo cual demuestra baja prioridad para resolver problemas y obstáculos que impiden a las mujeres participar en igualdad de condiciones con respecto a los hombres en el ámbito político (Herrera, 2010).

Finalmente, se deben tener en cuenta a las mujeres jóvenes de la sociedad, ya que estas tienen otras perspectivas con respecto al desarrollo cultural y social. Acosta y Garcés (2010) afirman que las mujeres jóvenes se muestran propensas a luchar por las pequeñas causas y por asuntos que se encuentren 
cercanos a su cotidianidad, tiene en cuenta que las luchas comienzan desde los escenarios familiares y poco a poco se convierten en necesidades globales.

Por su parte, Ahogado (2011) afirma que gran parte de las mujeres que han participado en la política en Colombia lo han logrado debido a otras condiciones sociales diferentes a las de su género, actuando como miembros de corporaciones, en su mayoría, las mujeres colombianas que participan en la política viven sujetas a lazos de dependencia y subordinación personal. Todo ello se concreta en la formación de una identidad independiente del género. De ahí que resalte algunas causas de esta escasa participación política de las mujeres en el país, entre ellas las barreras culturales, estereotipos y prejuicios sociales.

Así las cosas, se hacen visibles los factores que dificultan la participación femenina en el poder y la vida política: el juego político local caracterizado por redes políticas jerarquizadas y lógicas clientelistas regidas por altas inversiones de capital; la disposición del tiempo para participar en la política; la oposición de sus familias y el conflicto armado interno que afectan la participación política de las mujeres en las instancias locales. En los últimos años ha aumentado el número de mujeres que deben asumir la jefatura del hogar, aquello les resta oportunidades de participar en espacios de toma de decisiones.

Durango (2012) plantea que la Ley de Cuotas, en tanto acción afirmativa, representa un avance para la inclusión de un porcentaje de participación de las mujeres en los cargos de representación popular, cargos de los cuales han estado excluidas por siglos y la historia no data sobre ellas según sus participaciones en procesos de lucha. Con lo anterior, se evidencia que a pesar de que en su gran mayoría, la participación de las mujeres no ha sido efectiva, algunas instancias públicas sí las reconocen como sujetos políticos con derecho a participar en organizaciones o grupos en pos del bienestar, tanto de ellas como de la población en general.
Pinto (2013) ayuda a continuar con este argumento al señalar que es necesario resaltar los esfuerzos realizados por las mujeres históricamente con el fin de obtener reconocimiento como sujetos activos dentro de la sociedad; es notable que por medio de las declaraciones de derechos humanos y marcos legales se les ha otorgado más valor y reconocimiento, lo que ha posibilitado que tengan un espacio, aun así se deben esforzar para que su liderazgo no quede relegado.

Se debe mencionar que entre las causas por las cuales las mujeres no tienen un lugar representativo en la política colombiana se encuentran hechos como la violencia, conflicto armado, machismo político, quehaceres del ámbito privado, así como múltiples variables más que hacen de la mujer un sujeto que no participa en la toma de decisiones. Al respecto, Martínez (2014) indica que en Colombia la baja participación de las mujeres en los cargos de representación política se debe a varios factores, como el rezago de la cultura patriarcal que todavía predomina en las regiones, principalmente en el ámbito de lo político, a pesar de los logros alcanzados.

Del mismo modo, Garzón (2018) afirma que las mujeres enfrentan diversos obstáculos para participar en la vida política, las barreras estructurales a través de leyes e instituciones discriminatorias aún limitan las opciones de las mujeres para presentarse a la presidencia. En conclusión, las brechas de capacidad significan que las mujeres tienen menos probabilidades que los hombres de tener la educación, contactos y recursos necesarios para convertirse en líderes eficaces.

El costo social de la violencia de género incluye la inacción de la sociedad que no toma medidas para defender los derechos humanos de las mujeres $y$, por lo tanto, ignora lo que sucede cotidianamente y niega la discusión pública de estos delitos, su significación política y su reparación social. Sánchez y Rodríguez (2015) afirman que a pesar de que las 
mujeres se cataloguen como las principales víctimas del conflicto, gracias a su activa participación en diferentes organizaciones han incidido en las acciones de reivindicación para con ellas. Además, da cuenta de cómo las mujeres convergen en varios aspectos, lo cual permite un avance efectivo en su posicionamiento en escenarios políticos.

El objetivo de alcanzar una igualdad de hombres y mujeres al momento de afrontar las elecciones está pendiente y requiere de toda la atención política de los actores que disputan y participan en las elecciones. Torres (2016) plantea que en Colombia se han obtenido varios avances legislativos para la inclusión y participación de la mujer en cargos de elección popular. Sin embargo, esas leyes no generan la plena representación de intereses y derechos de las mujeres en los diferentes municipios o regiones del país, ya que dicha inclusión obedece a otras prácticas políticas tales como: bases comunitarias existentes en la localidad que permiten una votación suficiente para obtener un curul; reclutamiento por parte de partidos políticos; la labor como cuidadoras de votos de otros líderes políticos; y por último, reemplazos a algún candidato por orden de lista.

Así entonces, se evidencia que en la normatividad colombiana hay legislación que propone a la mujer como sujeto político, pero que esta no tiene cumplimiento efectivo en su totalidad. Al respecto, Martínez, Quintero, Londoño y Klimenko (2016) argumentan que en Colombia se ha avanzado significativamente a nivel legislativo en relación con la participación política de las mujeres, sin embargo, no es suficiente para que realmente se garanticen los derechos de igualdad y los espacios de participación política que estas reclaman aún desde diferentes escenarios y organizaciones sociales. Las mujeres han participado y trabajado en la mesa en Cooperación Internacional para el fortalecimiento y articulación de redes de mujeres a nivel local y departamental, además para la creación de un modelo de formación a posibles candidatas a elecciones populares.
Al indagar por los distintos intereses en juego en el fortalecimiento de los mecanismos de participación política efectiva de las mujeres, Burbano, Medina y Calvache (2016) plantean que la participación política de las mujeres deriva de dos tipos de intereses: los intereses prácticos que buscan la satisfacción de las necesidades derivadas de la desigualdad de género; y los intereses estratégicos que suponen reivindicaciones que pretenden transformar las relaciones sociales para que sean más equitativas. Según los autores es posible afirmar que la participación política es la capacidad de las mujeres de ser elegidas y de elegir a sus mandatarios, pero también, es la posibilidad de hacer valer sus derechos constitucionales fundamentales. De ahí que los programas de formación política, en su gran mayoría, tienen como objetivo central lograr que las mujeres se formen políticamente para que hagan parte de cargos de elección popular e incidan en las políticas públicas a favor de la mujer y conjuntamente, que desde las diferentes organizaciones sociales y comunitarias luchen por sus derechos con distintas formas de resistencia.

Un balance del estado de la participación en América Latina e igualdad de género ubica a Colombia como un país de escaso cumplimiento de estas condiciones. Según Barona y Mayor (2017), Colombia es el último país de América Latina en implementar acciones afirmativas dentro de sus legislaciones para garantizar la igualdad de género con respecto a las elecciones populares en el país, ya que la Ley 1475 del 2011 propone el principio de igualdad de género. Asimismo, se estableció el 30 \% de participación femenina en las listas de partidos y movimientos políticos para cargos de elección popular. No obstante, se encuentra que una vez entró en vigencia la Ley 1475 del 2011m hubo un aumento en la participación de las mujeres, más sin embargo, su representación sigue siendo insuficiente con respecto a las mujeres en otros países que logran ocupar un escaño, en comparación a la participación en listas de cada partido o movimiento político. 
Lo anterior da cuenta de que las figuras patriarcales ahondan en la poca o baja participación de las mujeres en esferas públicas, esto evidencia la violencia como uno de los ejes principales para la no participación de las mujeres en ámbitos decisivos, tanto a nivel local y regional como nacional. De acuerdo con lo planteado anteriormente, Yace (2017) afirma que la violencia inhibe la participación de las mujeres en la adopción de decisiones, tanto dentro del hogar como en el ámbito laboral, político, económico y social, por lo que influye directamente en su participación pública y por lo tanto, en el ejercicio de la ciudadanía.

Uno de los mecanismos para que las mujeres entren en el juego político, en algunas ocasiones, son las ayudas económicas por parte del Estado, estras ayudan a que se sientan más empoderadas, ya que cuentan con una ayuda económica, lo que les permite catalogarse como mujeres fuertes y partícipes en ámbitos sociales. López (2017) plantea que los programas de transferencia condicionada como Familias en Acción pueden aumentar la probabilidad de que las mujeres participen activamente en organizaciones civiles, votaciones y protestas. Sin embargo, los resultados arrojaron que, si bien hay evidencia de que se pueda aumentar la probabilidad de participar, esto ocurre solo en algunas organizaciones civiles y en las votaciones regionales principalmente, especialmente cuando son actividades que comprometen temas del bienestar de sus hogares y entorno.

Además, López (2017) añade que la participación femenina en acciones políticas depende de la ocupación, el nivel de ingresos y el estado civil, Así mismo, se deben tener habilidades necesarias para la subsistencia como tener empleo o ayudas estatales, esto conlleva que la mujer obtenga poder en las negociaciones que se dan tanto dentro como fuera del hogar. Es decir, la autonomía económica y el rol en el hogar parecen ser determinantes para la participación política femenina.
Finalmente, para concluir con los resultados de la revisión bibliográfica sobre participación política de las mujeres, en clave de sucesos próximos en la historia política de Colombia, Vargas (2017) menciona que uno de los hechos de participación política más relevante de las mujeres en Colombia es la subcomisión de género en los diálogos de Paz en la Habana, entre el Gobierno de Colombia y las Fuerzas Armadas Revolucionarias de Colombia. Tiene en cuenta que es importante alcanzar una integración de perspectivas de mujeres y así lograr que sigan adelantando procesos de construcción de paz desde sus organizaciones; el fin de la subcomisión de género era empoderar a las mujeres colombianas, sin hacer distinción por su origen o condiciones.

\section{Conclusiones}

Una de las primeras conclusiones luego de revisar la producción académica, radica en la necesidad de continuar en el país con la tradición de estudiar la participación política de las mujeres, pero en clave de estudios regionales y locales. Resulta pertinente conocer casos o prácticas adelantadas en espacios micro rurales, más, si uno de los hallazgos tiene que ver con la oportunidad de estudiar la participación en clave de representación en escenarios de paz territorial.

Con respecto a los estudios que indagan por las condiciones de las mujeres en escenarios de participación, el grueso de las investigaciones plantea que es desde el reconocimiento de las luchas por la reivindicación de derecho que se logran avances en términos normativos y participativos. Frente a este último punto -la participación política- la conclusión es que la participación es baja, que la legislación ha diseñado mecanismos para el ejercicio de este derecho, pero normas como la Ley de Cuotas no son efectivas para que las mujeres estén plenamente representadas o sean tomadas en cuenta en todos y cada uno de los escenarios de toma de decisión.

La siguiente conclusión está orientada a aquellas investigaciones que indagan por condiciones 
subjetivas que intervienen en la participación política. La participación de las mujeres también se evidencia en ámbitos culturales y sociales, además de los espacios políticos. Colombia debe consolidar una democracia fuerte para la participación política de todos los estamentos y grupos sociales, y evitar así fenómenos de discriminación y exclusión hacia las mujeres. Cabe resaltar que estas han estado en organizaciones y han luchado en pos de promover bienestar social e igualdad de condiciones para todos.

En Colombia resulta necesario fortalecer los escenarios de participación de las mujeres, estas se enfrentan de larga data a dinámicas de marginación y relegación debido a lógicas patriarcales. Se debe tener en cuenta que sus problemáticas se deben visibilizar y así mismo, tener espacios participativos para la colaboración con diferentes sectores. A pesar de que la Ley 1475 se implementó en el 2011, no evidencia ser eficiente pues no permite que en los espacios participativos haya igualdad entre hombres y mujeres.

\section{Referencias}

Acevedo, A., y Uribe, C. (2015). Mujeres y ciudadanía: Orígenes de un derecho y un debate sobre la participación política de las mujeres en el Gobierno. Revista Cientifica Guillermo de Ockham, 13(1), 109-116.

Acosta, G., y Garcés, A. (2010, enero-junio). Ámbitos y escenarios de participación juvenil en Medellín. Anagramas Rumbos y Sentidos de la comunicación, 8(16), 15-31.

Ahogado, T. (2011). Participación politica de las mujeres en cargos de elección popular en Bogotá: el concejo de Bogotá 2008-2011. Pontificia Universidad Javeriana.

Ariza, A. (2015). Participación de la mujer excombatiente en la vida política, social y cultural en Colombia. Pontificia Universidad Javeriana.
Barona, G., y Mayor, E. (2017). Participación y representación politica de las mujeres en el congreso de la república de Colombia. Universidad ICESI.

Batlle. M. (2016) Mujeres en el Congreso colombiano: un análisis a partir de la primera implementación de la Ley de cuota de género en las elecciones de 2014. Colombia internacional, 4(89). pp.17-49.

Beltrán, D. (2016). Transformaciones identitarias y participación politica de mujeres víctimas de violencia sexual en el conflicto armado colombiano. Pontificia Universidad Javeriana.

Bernal, A. (2006). ¿Qué es ganar y qué es perder en política?: los retos en la participación electoral. Revista Análisis Politico, (56), 72-92

Bernal, A. (2011). Elecciones parlamentarias de 2010: análisis de la inclusión de mujeres. Revista Colombia Internacional, 74, 89-118.

Burbano, G. C., Medina. L., y Calvache. C. (2018). Hacia la construcción de una propuesta de formación politica dirigida a mujeres. Universidad ICESI.

Burbano, G. C. (2016). Marco de fundamentación pedagógica de una propuesta de formación politica dirigida a mujeres pertenecientes a la organización para el desarrollo urbano y campesino. Pontificia Universidad Javeriana.

Caputto Silva, L. A. (2007). La mujer en Colombia: educación para la democracia y democracia en la educación. Revista Educación y Desarrollo Social, 2(1), 112-121.

Comisión Económica para América Latina y el Caribe (Cepal). (2014, agosto 29). Comisión Económica para América Latina y el Caribe [infografía]. http://wuww.cepal.org/es/infografias/ participacion-politica-de-las-mujeres 
Cortes, A., y Parra, G. (2008). Participación social y política: estudios de liderazgos femeninos en Bogotá y Cundinamarca. Revisit International journal of Psychological Research, 1, 40-48.

Dietrich, L. (2014). La «compañera política»: mujeres militantes y espacios de «agencia» en insurgencias latinoamericanas. Colombia Internacional, (80), 83-133.

Durango. G. (2012). Los movimientos sociales desde la perspectiva de las mujeres y pueblos indígenas. Un análisis desde la jurisprudencia de la Corte Constitucional colombiana. Revista de Derecho, (37), 111-130.

Escobar, K. (2014). Dinámica de la participación politica de las mujeres en el Congreso de la República de Colombia 1998-2014. Universidad del Valle.

Fuentes Vásquez, L. Y. (2009). Políticas públicas para la diversidad en Bogotá: la incidencia de las mujeres. Revista Nómadas, (30), 148-162.

Garzón, A. (2018). La mujer y el ejercicio politico: Clientelismo, redes politicas. Pontificia Universidad Javeriana.

Gutiérrez, A. (2015). Papel y empoderamiento institucional de la mujer en la organización del partido conservador colombiano, 2000-2017. Universidad Colegio Mayor Nuestra Señora del Rosario.

Gutiérrez, F. (2002). Degradación o cambio. Evolución del sistema político colombiano. Revista Estudios Politicos, 21, 163-176.

Hernández, J. (2014). La construcción periodística de la participación de las mujeres en política y en el crimen organizado. Ánfora, 2ł37), 37-60.
Herrera, A. (2010). Las Mujeres Frente a la Violencia de Género y la Participación Politica en Risaralda. Universidad Católica de Risaralda.

Hoyos G, D., y Nieto G, A. (2017). Procesos organizativos de mujeres y víctimas del conflicto armado y sus relaciones con la democracia local en el Oriente Antioqueño. Desafios, (29-1), 139-175.

Lamus Canavate. D. (2008), El lugar político de las mujeres en el movimiento negro/afrocolombiano. Reflexión Politica, 1020), pp. 236-257.

Lodoño, C. (2007). Políticas estatales para la equidad y la participación política de la mujer. Revista prospectiva, 2, 56-76.

López, L. (2017). Efecto del acceso a programas sociales en la participación politica de las mujeres. Universidad del Rosario.

Lora, R. (2015). La participación de la mujer en la politica colombiana entre las acciones afirmativas y la ley 1475 de 2011. Universidad Católica de Colombia.

Lozano, C., y Molina, E. (2014). La ley de cuotas como mecanismo eficiente en la participación política de las mujeres. Justicia Juris, 1022), 83-94.

Machado Pardo, L. (2018). El papel de la mujer en la independencia colombiana. Cultura Latinoamericana, 271), 74-98. https://doi. org/10.14718/CulturaLatinoam.2018.27.1.4

Martínez, B. (2014). Mujer, Educación y Participación Politica en el Quindío. Universidad San Buenaventura.

Martínez Flórez, L., Quintero Díaz, J., Londoño López, A., y Klimenko, O. (2016). La participación política de la mujer en la Comuna 4 Aranjuez, Medellín, Colombia, Revista Katharsis, (22), 165-195. 
Montoya, A. (2009). Mujeres y ciudadanía plena, miradas a la historia jurídica colombiana. Opinión Jurídica, 8(16), 137-148.

Morales. (2016). Análisis de la participación politica de la mujer indigena por circunscripción especial para senado 1991-2014. mecanismos, alcances y límites. Universidad Colegio Mayor Nuestra Señora del Rosario.

Murray, P. (2009). Mujeres, género y política en la joven república colombiana: una mirada desde la correspondencia personal del General Tomás Cipriano de Mosquera, 1859-1862. Historia Crítica, (37), 54-71.

Nemeguen C., y Tannia, J. (2012), El ejercicio politico de las mujeres. Una contribución a la resignificación del territorio urbano popular. Universidad Pedagógica Nacional.

Organización de las Naciones Unidas (ONU). (2012, marzo 19). Resolución aprobada por la Asamblea General el 19 de diciembre de 2011 [sobre la base del informe de la Tercera Comisión (A/66/455 y Corr.1)] 66/130. La participación de la mujer en la politica. Naciones Unidas. http://wuww. un.org/ga/search/view_doc.asp?symbol=A/ RES/66/1308referer=htto://hq.unw omen.org/ en/what-we-do/leadership-and-politicalparticipation\&lang=S

Parrado M, E., y Isidro, L. (2014). La paradoja hipócrita. Problematización de la participación política de la mujer Misak. Colombia Internacional, (80), 135-170.

Patiño, E. (2011). Género, participación y políticas públicas en Colombia: espacios en construcción. Plumilla Educativa,
8(2), 323-339. $\quad$ https://doi.org/10.30554/ plumillaedu.8.502.2011

Pinto, B. (2013). Los estereotipos, una limitante para que las mujeres ejerzan cargos en la alta gerencia. Universidad Militar Nueva Granada.

Pérez. A., y Cortes, M. (2012). Políticas públicas para la mujer en Colombia: La doble condición de madre y trabajadora en la legislación del siglo XX. Entramado, 8(1), pp. 72-88.

Rueda, M. (2016). Participación politica de las mujeres en la Unión Patriótica memorias de quienes construyen partido. Universidad Pedagógica.

Ruiloba, M. (2013). Liderazgo político y género en el siglo XXI. Revista Entramado, (17), 142-155.

Salcedo-Díaz, L., Silvera-Sarmiento, A., y GarcésGiraldo, L.F. (2017). Análisis de la obtención del derecho al voto como factor que incide en cambios generados en los movimientos de mujeres en Colombia. Revista Latinoamericana de Estudios de Familia, 9, 117-130.

Sánchez Mora, M., y Rodríguez Z. (2015). Acciones colectivas de las organizaciones de mujeres por la paz en Colombia. Revista de Paz y Conflictos, 8(2), 149-177.

Torres, A. (2016). Participación politica de las mujeres en el ámbito local de la ciudad de Bogotá. Pontificia Universidad Javeriana.

Triana, G. (2016). El efecto de la cuota de género en Colombia: un panorama sobre la participación politica femenina durante las elecciones legislativas de 2014. Universidad Colegio Mayor Nuestra Señora del Rosario. 
Tula, I. (2015). Mujeres y política, Un panorama sobre la adopción de las cuotas de género y sus efectos en América Latina y Colombia. Observatorio de Políticas, Ejecución y Resultados de la Administración Pública, (16), 9-33.

Uribe Ochoa, C. (2015). Presencia femenina en la Asamblea Departamental de Santander y en el Concejo Municipal de Bucaramanga durante el Frente Nacional. Folhmyp, (3), 3-21. https://revistas. pedagogica.edu.co/index.php/FHP/article/view/3287

Valderrama, M. (2018). De la lucha armada a la politica: una visión de las mujeres. Pontificia Universidad Javeriana.
Vargas, L. M. (2017). La participación de la mujer en la subcomisión de género. Universidad Militar Nueva Granada. htto://hdl.handle. net/10654/16571.

Vos Obeso, R. (2004). Balance histórico de la participación política de las mujeres colombianas en el siglo XX. Historia Caribe, /U9), pp. 7-22.

Yace, I. (2017). Participación politica de las mujeres en condición de desplazamiento en el consejo comunitario local en el municipio de López de Micay. Pontificia Universidad Javeriana. 